\title{
(2) OPEN ACCESS \\ Fighting a common enemy: a catalyst to close intractable safety gaps
}

\author{
Hardeep Singh (1) , Dean F Sittig, ${ }^{2}$ Tejal K Gandhi ${ }^{3}$
}

${ }^{1}$ Center for Innovations in Quality, Effectiveness and Safety, Michael E. DeBakey Veterans Affairs Medical Center and Baylor College of Medicine, Houston, Texas, USA ${ }^{2}$ School of Biomedical Informatics, University of Texas Health Science Center, Houston, Texas, USA

${ }^{3}$ Press Ganey Associates LLC, Boston, Massachusetts, USA

\section{Correspondence to} Dr Hardeep Singh, Michael E. DeBakey Veterans Affairs Medical Center (MEDVAMC), Center for Innovations in Quality, Effectiveness and Safety (152), 2002 Holcombe Boulevard, Houston, TX 77030, USA; hardeeps@bcm.edu

Received 23 April 2020 Revised 16 June 2020 Accepted 19 June 2020 Published Online First 16 July 2020

\section{Check for updates}

(c) Author(s) (or their employer(s)) 2021. Re-use permitted under CC BY-NC. No commercial re-use. See rights and permissions. Published by BMJ.

To cite: Singh $H_{\text {, Sittig DF }}$ Gandhi TK. BMJ Qual Saf 2021:30:141-145.
Since March 2020, the world has faced a singular threat: COVID-19. The shared commitment and responsibility uniting everyone within and outside of healthcare to bend the COVID-19 curve has been unparalleled. Prior to the pandemic, this type of shared commitment has been discouragingly lacking for other major healthcare concerns such as patient safety. ${ }^{1}$ Reasons for this include organisational leaders who are incentivised to focus on activities essential for reimbursement and quality measurement rather than those involving the promotion of safety culture and implementation of systemsbased approaches to improve safety, compounded by lack of clear ownership and accountability to solve long-standing safety challenges. ${ }^{23}$

The COVID-19 pandemic is leading to several ongoing impacts on the healthcare delivery system, ${ }^{4}$ many of which have patient safety implications and will be quantified in future work. We are witnessing negative effects from delays in care from patients not seeking (or unable to seek) healthcare, patients with complex chronic conditions not having ongoing ambulatory care and new types of diagnostic errors. ${ }^{5}$ However, we are also witnessing some early short-term positive effects in selected safety areas where the COVID-19 pandemic has provided a new glimmer of hope. As of now, several changes have occurred in at least three safety-related domains: (1) adoption of key attributes of safety culture (transparency, communication and collaboration); (2) rapid implementation of safety practices to care for the previously often neglected healthcare workforce; and (3) use of state-of-the-art health information technology (IT) to improve the safety of patients and clinicians within the healthcare delivery system. Even though currently only temporary and not universal, the rapid adoption of these strategies demonstrates the potential of diverse stakeholders with competing interests to rally together against a common enemy, in this case a deadly virus.

Bringing about long-term changes and improvement of patient safety outcomes requires dedicated sustainment efforts and continuous evaluation to minimise unintended consequences. Safety professionals are now uniquely positioned to contribute to the know-how of how to address organisational change to improve care, reduce variations and overcome long-term inertia. ${ }^{6-8}$ In this Viewpoint, we present some examples for optimism resulting from unifying against a common enemy and discuss why early transformations in three safety domains should be harvested to create permanent mechanisms to target another common enemypreventable harm-in the next decade. In an effort to address long-standing safety challenges, we posit that harvesting lessons learned from early COVID-19 related transformations could sustainably 'accelerate the curve' of Diffusion of Innovations ${ }^{9}$ such that more organisations (so-called 'laggards' and 'late majority' that are delayed in adoption of best practices) will more rapidly adopt useful safety practices and innovations going forward (figure 1).

\section{OVERCOMING INERTIA FOR SAFETY CULTURE}

Despite being a major focus of safety efforts, a safety culture where everyone feels comfortable speaking up about errors and near misses without fear of unjust punishment has remained frequently unachievable. ${ }^{10}$ Healthcare organisations have not prioritised transparency and 


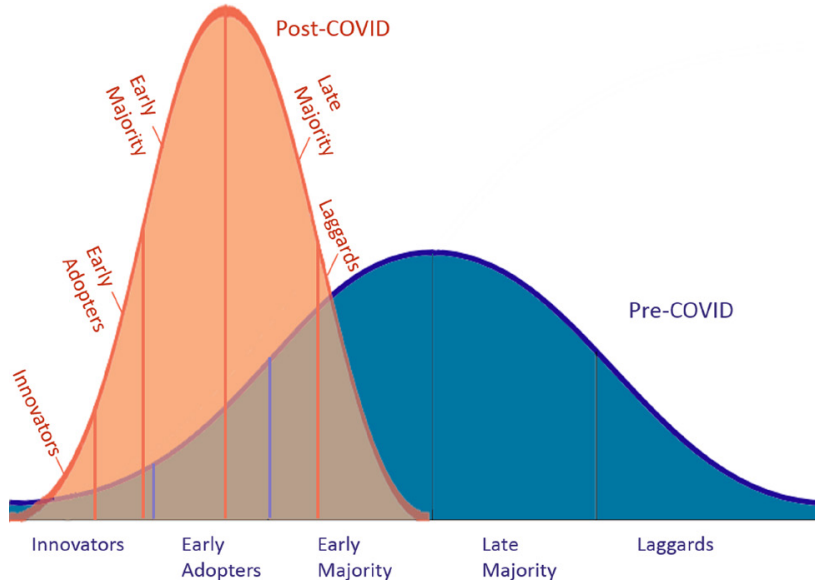

Figure 1 Potential use of COVID-19 as a catalyst to accelerate the Diffusion of Innovations curve for patient safety.

learning. Culture change requires strong leadership for creation and sustainability, but progress has been frustratingly slow. ${ }^{11}$ In 2018, for example, less than half of respondents on organisational culture surveys report working in a non-punitive environment. ${ }^{12}$

So why should this be an opportune time to catalyse sustainable culture change? During the current pandemic, we are witnessing previously unimaginable rapid shifts in culture-related practices and focus from leadership. Fighting the pandemic has resulted in major changes in healthcare delivery and exposed several health system vulnerabilities. Certain characteristics of healthcare organisations that struggle to improve quality, ${ }^{13}$ including poor organisational culture, lack of a cohesive mission and vision and dysfunctional external relations, all have been tested by COVID-19. Leaders have realised that in order to successfully battle this disease, they need to focus on culture and transparency to drive organisational change. Many organisational leaders are communicating daily with urgency about safety and much more visibly through teleconferences, daily huddles and safety rounds, often getting frequent input from the frontlines about concerns. ${ }^{14} 15$ Incident command structures also facilitated rapid decision making and breaking down silos. In addition, at the individual clinician level, far less resistance occurred for rapid deployment of new protocols and standardisation of care processes.

Transparency also has rapidly become the norm with daily communications about census, personal protective equipment (PPE) availability, numbers of cases and deaths not just internally but also to the public. Transparency across organisations has hit a new high, with clinicians and hospital system leaders working together regionally and nationally to rapidly share internal data, concerns, challenges and best practices. ${ }^{15} 16$ For instance, the world's largest medical centre complex, Texas Medical Center in Houston, Texas, is providing data to the public on infection rates, actions to increase patient capacity, ICU bed availability modelling, ventilator availability, PPE needs and current COVID-19 hospitalisations and deaths involving all major local hospitals. ${ }^{17}$ Data on healthcare indicators, and safety in particular, has rarely been shared so openly yet now this is becoming a basic, daily expectation.

These leadership behaviours could be the impetus for organisations to continue moving towards a highreliability organisation culture that facilitates transparency, communication, collaboration, non-punitive reporting and speaking up. These behaviours are paramount for creating a culture of safety and high reliability and illustrate at least 3 of the 5 principles of high-reliability that could be leveraged for longterm sustained impact: (1) sensitivity to operations with 'heightened awareness of the state of relevant systems and processes'; (2) deference to expertise by valuing insights from staff with safety knowledge; and (3) increasing resilience by proactively preparing for possible system failures. ${ }^{18}$

Nevertheless, not all organisations have witnessed the same level of transparency and/or witnessed short-term effects on culture. ${ }^{19}$ In addition, there are anecdotes of safety reporting rates going down during the crisis, possibly due to time pressures or lack of psychological safety. Going forward, we recommend efforts to sustain early positive momentum and to reduce the risk of unintended consequences. For example, organisations should implement safety culture measurement and continue daily huddles, leadership rounding and transparent communication of both positive and negative events within and across organisations. For all organisations-those who have been leaders on implementation and those who are lagging - these behaviours and strategies will need to be sustained to ensure that a high reliability culture promotes learning from failures and safety improvements across the board. ${ }^{20}$ This is how more organisations could achieve 'early majority' status on an 'accelerated' Rogers' Diffusion of Innovations curve for patient safety.

\section{PRIORITISING WORKFORCE SAFETY}

Even before the pandemic, healthcare was more dangerous than mining, manufacturing or construction, with burnout and suicide rates much higher than the general population. ${ }^{21} 22$ Yet, attention to the physical and emotional well-being of the workforce has been difficult to gain other than recently for burnout. ${ }^{22}$ Support systems are few, and previously healthcare workers have often kept working due to organisational expectations even when symptomatic with infections. ${ }^{23}$ Other forms of injury and illness, resulting from slips, needle-sticks, falls, moving patients and infections such as influenza and Methicillin-resistant Staphylococcus aureus (MRSA) ${ }^{24}$ lead to significant days lost from work and sometimes long-term sequelae. However, they 
are seen as part of doing business by leaders even though it is clear that workforce safety is a precondition for patient safety. Notably, the majority of hospital boards often do not even routinely review workforce injury rates. ${ }^{25}$

The COVID-19 pandemic has rapidly brought discussions of worker physical safety front and centre for hospital leaders, particularly with the challenges of obtaining PPE. Workforce infection rates are now being tracked diligently with great amounts of effort focused on procuring PPE, protocols for use, as well as training and monitoring staff on donning and doffing. Multiple efforts have been implemented in very short time frames to address concerns about the emotional and psychological harms to the workforce. ${ }^{26}{ }^{27}$ Many leaders are purposefully communicating about how important it is to get help dealing with anxiety, stress and uncertainty, and further making sure this is not viewed as a sign of weakness. Some organisations are creating well-being teams with robust staff support mechanisms. ${ }^{28}$ Counselling has become readily available and is no longer seen as a stigma. Buddy systems to help providers check in on each other's well-being, hotlines to ask for help and personal well-being strategies are being implemented. ${ }^{29}$ Finally, the US government has relaxed regulations ${ }^{30}$ allowing healthcare organisations to provide benefits and support to their workers in the form of food, childcare issues and laundry services, and WHO has selected 'Health Worker Safety: A Priority for Patient Safety' as the theme for World Patient Safety Day 2020.

However, not all organisations have done a positive job with workforce safety. Thousands of healthcare workers have been affected worldwide, and many have died from COVID-19 as a result due to lack of availability of adequate personal protection. In addition, some organisations targeted workers who spoke up against poor safety practices, ${ }^{31}$ and there are reports of trainee mistreatment. ${ }^{32}$ Nurses have been particularly affected ${ }^{33}$ and so have nursing aides and support workers in nursing homes. ${ }^{34}$ For instance, in the midst of inadequate staffing and PPE, nurses have been exploited and/or silenced with reports that certain hospitals suspended nurses trying to fundraise for PPE and forced them to work while they showed COVID-19 symptoms if tests were negative. ${ }^{33}$

Even though not all organisations are currently nurturing workforce physical and psychological wellbeing similarly, the early positive efforts should inspire others and continue beyond this current crisis, as physical and emotional safety concerns preceded this crisis and will also exist beyond it. ${ }^{35}$ It will also be important to make lasting changes to support the workforce and address neglected areas across the continuum such as safety in nursing homes. ${ }^{36}$ Only then can we ensure that these irreplaceable human resources are available when most needed.
REMOVING BARRIERS TO USING HEALTH IT TO IMPROVE SAFETY

Over the last decade, despite billions being spent on implementation of health IT, use of health IT to improve patient safety remains suboptimal. ${ }^{37}$ Technology applications, including electronic health records (EHRs) and the digital data they capture, have not been adequately used to either predict or reduce preventable harm. ${ }^{38}$ EHRs have emerged as a major source of clinician burnout with potential negative effects on patient safety. ${ }^{22}$ Involved stakeholders (clinicians, healthcare organisations, vendors, government agencies and regulators) have tended to attribute failures to one another. ${ }^{39}$ Yet over the past few months, we have seen unparalleled cooperation between these stakeholders leading to substantive progress in removing previously intractable barriers and allowing technology to help clinicians do their work and improve quality and safety.

For example, prior to the COVID-19 crisis, telemedicine, in all its incarnations over the last 25 years, ${ }^{40}$ was limited in USA because Centers for Medicare \& Medicaid Services and other insurers would not pay for it in most situations, physicians were limited to providing services in states they were licenced in and overly restrictive Health Insurance Portability and Accountability Act regulations limited the types of hardware and software for connecting physicians and patients. ${ }^{41}$ Within a few days, these non-technical barriers were removed ${ }^{42}$ when it became apparent that telemedicine enabled many physicians to continue to care safely for their patients while eliminating risks of virus transmission in the waiting or exam rooms. This led to organisations and clinicians, often resistant to major changes in clinical workflow, implementing telemedicine rapidly and efficiently. ${ }^{43}$ In addition to creating telehealth solutions, EHR vendors have partnered with healthcare organisations in new ways-working expeditiously on sharing records more broadly and implementing new screening tools, predictive analytics, charting templates, order sets, reporting tools and dashboards and access to data streams for research. ${ }^{44}$ Finally, the unique collaboration between Google and Apple to enhance the Bluetooth capabilities of their mobile phone operating systems to enable anonymous contract tracing between individuals infected with COVID-19 is astonishing on technical, social and legal fronts. ${ }^{45}$

Nevertheless, removing barriers to development and use of health IT interventions, be they telemedicine or apps, is not without risk. While telemedicine has been around for several decades, its benefits are still being studied. Increased use of telemedicine may be good in the short term, but its safety and long-term workforce implications are unclear and warrant evaluation. For instance, it could lead to less need for healthcare workers with subsequent job losses and increased adverse events such as diagnostic and management 
errors. Furthermore, widespread use of telemedicine and emerging apps has the potential to lead to data privacy breaches, billing fraud and inappropriate care. While having the two largest technology companies working together is also not a fail-safe solution, without such cooperation, widespread contact tracing may not be possible. During times of public health emergencies, we may need to err on the side of collecting and using data to protect lives while cautiously working to prevent the potential for abuse by developing new rules and regulations against the misuse of these data. ${ }^{46}$ This is admittedly a difficult trade-off to manage. ${ }^{47}$

In the past, without this common enemy, this shared responsibility and technical collaboration between vendors and with vendors and the clinical world has been unachievable even in the midst of long-standing safety concerns about poor usability and interoperability. ${ }^{39}$ Now that there is precedent for change, it is time to remove sociotechnical barriers and use IT and related data science solutions to predict and reduce harm beyond the current crisis.

\section{CONCLUSION}

Rapid care delivery transformation has been a silver lining in recent months. As the COVID-19 pandemic subsides, healthcare will still be left with the chronic and widespread public health crisis of preventable patient and workforce harm. Similar to the virus, safety issues affect all healthcare stakeholders, including patients and clinicians, either physically, financially or emotionally. The existential threat that every healthcare stakeholder is facing during the COVID-19 crisis has galvanised a response across multiple stakeholders (government, health systems leaders and clinicians, industry and the general public to name a few) with promising strides that could improve future safety culture, prioritise workforce safety and remove barriers to use of IT to improve safety. Harvesting lessons from these transformations, continuous evaluation of promising early changes and dedicated sustainment efforts could accelerate the curve of Diffusion of Innovations to promote more rapid and successful spread of safety strategies and innovations. Efforts must be made now to fully catalyse this momentum to overcome decades of slow progress in reducing preventable medical harm.

Twitter Hardeep Singh @HardeepSinghMD, Dean F Sittig $@$ DeanSittig and Tejal K Gandhi@TGandhi_Safety

Contributors Each author contributed to the writing of the manuscript.

Funding Dr Singh is funded in part by the Houston Veterans Administration (VA) Health Services Research and Development (HSR\&D) Center for Innovations in Quality, Effectiveness, and Safety (CIN13-413), the VA HSR\&D Service (CRE17-127 and the Presidential Early Career Award for Scientists and Engineers USA 14-274), the VA National Center for Patient Safety, the Agency for Healthcare Research and Quality (R01HS27363), the CanTest Research Collaborative funded by a Cancer Research UK Population Research Catalyst award (C8640/A23385) and the Gordon and Betty Moore Foundation.

Disclaimer The views expressed in this article do not represent the views of the U.S. Department of Veterans Affairs or the United States government.

Competing interests Dr Gandhi is an employee of Press Ganey Associates LLC.

Patient consent for publication Not required.

Provenance and peer review Not commissioned; externally peer reviewed.

Data availability statement There are no data in this work.

Open access This is an open access article distributed in accordance with the Creative Commons Attribution Non Commercial (CC BY-NC 4.0) license, which permits others to distribute, remix, adapt, build upon this work noncommercially, and license their derivative works on different terms, provided the original work is properly cited, appropriate credit is given, any changes made indicated, and the use is noncommercial. See: http://creativecommons.org/licenses/by-nc/4. $0 /$.

\section{ORCID iD}

Hardeep Singh http://orcid.org/0000-0002-4419-8974

\section{REFERENCES}

1 Bates DW, Singh H. Two decades since to err is human: an assessment of progress and emerging priorities in patient safety. Health Aff 2018;37:1736-43.

2 Wachter RM. Patient safety at ten: unmistakable progress, troubling gaps. Health Aff 2010;29:165-73.

3 Jarrett MP. Patient safety and leadership: do you walk the walk? J Healthc Manag 2017;62:88-92.

4 Berwick DM. Choices for the "New Normal". JAMA 2020;323:2125-6.

5 Gandhi TK, Singh H. Reducing the risk of diagnostic error in the COVID-19 era. J Hosp Med 2020;15:363-6.

6 Staines A, Amalberti R, Berwick DM, et al. COVID-19: patient safety and quality improvement skills to deploy during the surge. Int J Qual Health Care 2020. doi:10.1093/intqhe/ mzaa050. [Epub ahead of print: 13 May 2020].

7 La Regina M, Tanzini M, Fineschi V, et al. Responding to COVID-19: the experience from Italy and recommendations for management and prevention [Corrected Proof]. Int J Qual Health Care 2020. [Epub ahead of print: 19 May 2020].

8 Albolino S, Dagliana G, Tanzini M, et al. Human factors and ergonomics at time of crises: the Italian experience coping with COVID19. Int J Qual Health Care 2020. doi:10.1093/intqhe/ mzaa049. [Epub ahead of print: 13 May 2020].

9 Rogers E. Diffusion of innovations. 5th edn. New York: Free Press, 2003.

10 Basson T, Montoya A, Neily J, et al. Improving patient safety culture: a report of a multifaceted intervention. J Patient Saf 2018. doi:10.1097/PTS.0000000000000470. [Epub ahead of print: 09 Feb 2018].

11 National Patient Safety Foundation. Free from harm: accelerating patient safety improvement fifteen years after to err is human. Boston, MA, 2015.

12 Famolaro T, Yount ND, Hare R. Hospital survey on patient safety culture: 2018 user database report. Surveys on patient safety culture. Rockville, MD, 2018.

13 Vaughn VM, Saint S, Krein SL, et al. Characteristics of healthcare organisations struggling to improve quality: results from a systematic review of qualitative studies. BMJ Qual Saf $2019 ; 28: 74-84$. 
14 Ensuring Healthcare Safety Throughout the COVID-19 Pandemic. US Health and Human Services Office of the Assistant Secretary for Preparedness and Response's Technical Resources, Assistance Center, \& Information Exchange (ASPR TRACIE); US Health and Human Services /FEMA COVID-19 Healthcare Resilience Task Force, 2020. Available: https:// psnet.ahrq.gov/issue/ensuring-healthcare-safety-throughoutcovid-19-pandemic

15 Dudley J. COVID-19 caregiver collaborative: practical insights and notes from the field. Press Ganey, 2020.

16 Looi M-K, Coombes R. Risky business: lessons from covid-19. BMJ 2020;369:m2221.

17 Texas Medical Center. Current COVID-19 related patients through TMC system, 2020. Available: https://www.tmc. edu/coronavirus-updates/current-covid-19-related-patientsthrough-tmc-system/

18 Weick KE, Sutcliffe KM. Managing the unexpected: sustained performance in a complex world. 3rd edn. San Franciso: Jossey-Bass, 2015.

19 Klein M, Landman B. Mount Sinai Hospital leaders holed up in Florida vacation homes during coronavirus crisis (2020-0328). New York Post 2020.

20 Chassin MR, Loeb JM. High-reliability health care: getting there from here. Milbank Q 2013;91:459-90.

21 Occupational Safety and Health Administration. Understanding the problem - Worker safety in hospitals, 2020. Available: https://www.osha.gov/dsg/hospitals/understanding problem.html

22 National Academies of Sciences, Engineering, and Medicine. Taking action against clinician burnout: a systems approach to professional well-being. Washington, DC: The National Academies Press, 2019.

23 Szymczak JE, Smathers S, Hoegg C, et al. Reasons why physicians and advanced practice clinicians work while sick: a mixed-methods analysis. JAMA Pediatrics 2020;169:815-21.

24 Occupational Safety and Health Administration. Infectious diseases, 2020. Available: https://www.osha.gov/SLTC/ healthcarefacilities/infectious_diseases.html

25 McGaffigan PA, Ullem BD, Gandhi TK. Closing the gap and raising the bar: assessing board competency in quality and safety. Jt Comm J Qual Patient Saf 2017;43:267-74.

26 Wu AW, Connors C, Everly GS. COVID-19: peer support and crisis communication strategies to promote institutional resilience. Ann Intern Med 2020;172:822-3.

27 Cai H, Tu B, Ma J, et al. Psychological impact and coping strategies of frontline medical staff in hunan between January and March 2020 during the outbreak of coronavirus disease 2019 (COVID-19) in Hubei, China. Medical Science Monitor 2020:26.

28 Adibe B, Perticone K, Hebert C. Creating wellness in a pandemic: a practical framework for health systems responding to COVID-19. NEJM Catalyst 2020.

29 Berg S. Peer support program strives to ease distress during pandemic, 2020. Available: https://www.ama-assn.org/practicemanagement/physician-health/peer-support-program-strivesease-distress-during-pandemic

30 Center for Medicare and Medicaid Services. Additional background: sweeping regulatory changes to help U.S. healthcare system address COVID-19 patient surge, 2020.

Available: https://www.cms.gov/newsroom/fact-sheets/ additional-backgroundsweeping-regulatory-changes-help-ushealthcare-system-address-covid-19-patient

31 Gallegos A. Hospitals muzzle doctors and nurses on PPE, COVID-19 cases. Medscape 2020.

32 Gibson A. COVID-19 crisis exposes resident abuse, 2020. Available: https://www.webmd.com/lung/news/20200428/ covid-19-crisis-exposes-resident-abuse

33 Garber J. Protecting nurses during the COVID-19 pandemic - Lown Institute, 2020. Available: https://lowninstitute.org/ protecting-nurses-during-the-covid-19-pandemic/

34 Mueller B. On a Scottish Isle, nursing home deaths expose a Covid-19 scandal. The new York times: 20200525, 2020. Available: https://www.nytimes.com/2020/05/25/world/europe/ coronavirus-uk-nursing-homes.html

35 Dzau VJ, Kirch D, Nasca T. Preventing a parallel pandemic a national strategy to protect clinicians' well-being. $\mathrm{N} \mathrm{Engl} \mathrm{J}$ Med Overseas Ed 2020.

36 Abbasi J. "Abandoned" nursing homes continue to face critical supply and staff shortages as COVID-19 toll has mounted. JAMA 2020.

37 Singh H, Sittig DF. Measuring and improving patient safety through health information technology: the health it safety framework. BMJ Qual Saf 2016;25:226-32.

38 Murphy DR, Meyer AN, Sittig DF, et al. Application of electronic trigger tools to identify targets for improving diagnostic safety. BMJ Qual Saf 2019;28:151-9.

39 Sittig DF, Belmont E, Singh H. Improving the safety of health information technology requires shared responsibility: it is time we all step up. Healthc 2018;6:7-12.

40 Hersh WR, Helfand M, Wallace J, et al. Clinical outcomes resulting from telemedicine interventions: a systematic review. BMC Med Inform Decis Mak 2001;1:5.

41 Gabriel MH, Jones EB, Samy L, et al. Progress and challenges: implementation and use of health information technology among critical-access hospitals. Health Aff 2014;33:1262-70.

42 Services CfMM. Medicare telemedicine health care provider fact sheet, 2020. Available: https://www.cms.gov/newsroom/ fact-sheets/medicare-telemedicine-health-care-provider-factsheet

43 Wosik J, Fudim M, Cameron B, et al. Telehealth transformation: COVID-19 and the rise of virtual care. J Am Med Inform Assoc 2020;27:957-62.

44 Reeves JJ, Hollandsworth HM, Torriani FJ, et al. Rapid response to COVID-19: health informatics support for outbreak management in an academic health system. J Am Med Inform Assoc 2020;27:853-9.

45 Apple. Privacy-preserving contact tracing - Apple and Google, 2020. Available: https://www.apple.com/covid19/ contacttracing

46 Mello MM, Wang CJ. Ethics and governance for digital disease surveillance. Science 2020;368:951-4.

47 Sittig DF, Singh H. COVID-19 and the need for a national health information technology infrastructure. JAMA 2020. doi:10.1001/jama.2020.7239. [Epub ahead of print: 18 May 2020]. 